\title{
Abstracts of Posters
}




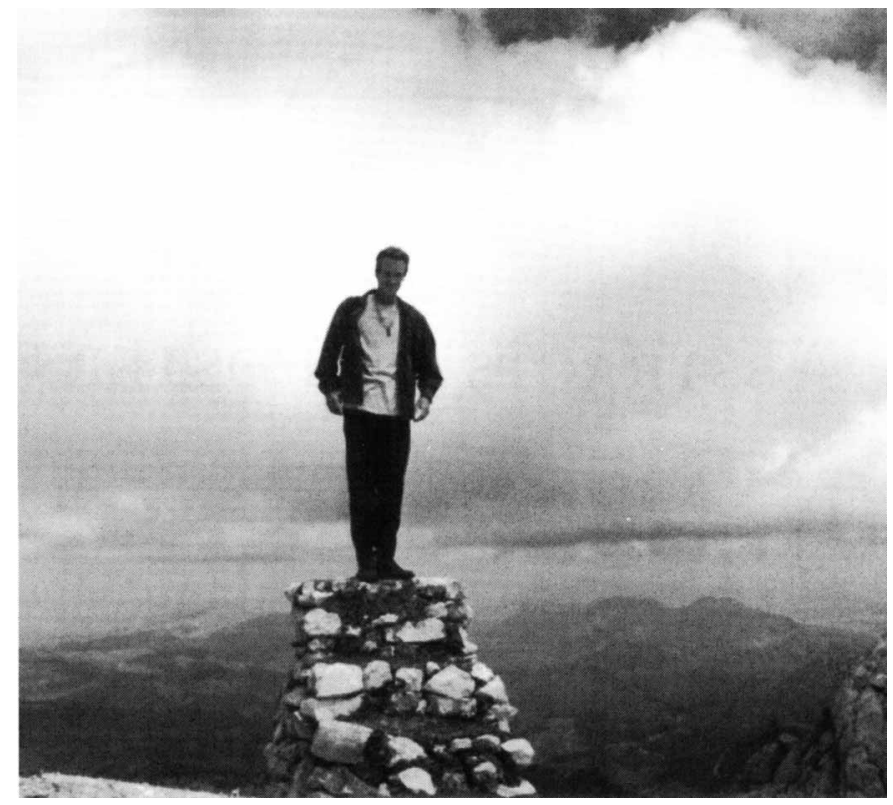

Jacco van Loon, known in some circles as der Fliegende Holländer, prepares to test his wings.

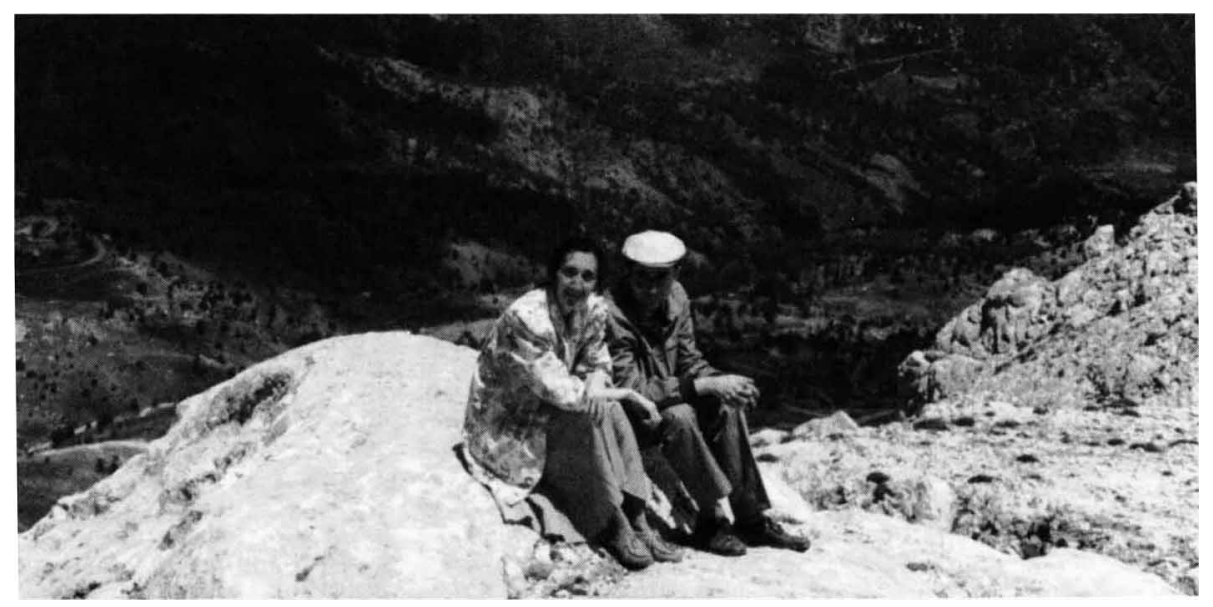

Tsitsino and Irakli Simonia, from the Republic of Georgia, feel right at home in the impressive scenery of the Toros mountains. 
POSTERS

\section{V and R Observations of Two Carbon Stars: UX Dra and RY Dra}

\section{HASAN AK ${ }^{1}$, BERAHITDIN ALBAYRAK ${ }^{1}$, ZEKİ ASLAN ${ }^{2}$, OSMAN DEMIRCAN ${ }^{1}$, ZEKERIYA MÜYESSEROḠLU ${ }^{1}$, SACIT ÖZDEMIR ${ }^{1}$, and KUTLUAY YÜCE ${ }^{1}$}

1 Ankara University Observatory, Ankara, Turkey

2 Akdeniz University, Antalya, Turkey

The carbon stars UX Dra and RY Dra, classified as semiregular variables, were observed photometrically in the $V$ and $R$ filters at the Ankara University Observatory during the period between April 1995 and March 1996. Altogether $\sim 19$ hours of observations on 7 nights for UX Dra and $\sim 11$ hours of observations on 4 nights for RY Dra were used in search of both short-term and long-term light variations.

UX Dra was at maximum light in January 1996 and its brightness decreased by $0.16 \pm 0.02 \mathrm{mag}$ in both filters in about 100 days. The minimum light occurred in April 1996. The light variation is not symmetrical. The period of light variation cannot be less than about 200 days. This means the lengthening of the period is about 1.5 day per year, which confirms Vetesnik's (1983, IBVS No. 2329) finding. The $V-R$ color curve follows a trend similar to the light curves, i.e. the star is hotter at maximum. Two 6.5 hour continuous observations show that UX Dra exhibits short-term light variations of up to $0.03 \mathrm{mag}$ in both filters.

RY Dra was also at maximum light in January 1996 and it faded by about $0.16 \pm 0.02 \mathrm{mag}$ in $V$, and by $0.10 \pm 0.02 \mathrm{mag}$ in $R$, in about 20 days. Due to gaps in our observations, the period of light variation cannot be estimated. As in the case of UX Dra, the $V-R$ color curve follows a trend similar to the light variation. No significant short-term light variation was detected in our observations. 